\title{
The Effect of Variation of the Layout Angle of Plane Figures on their Apparent Sizes for Rectangles with Different Proportions
}

\author{
Takeshi KINOSHITA and Yumie FUKUDA
}

Graduate School of Science and Engineering, Yamaguchi University, 2-16-1 Tokiwadai, Ube 755-8611, Japan

\begin{abstract}
In visual communication media, various symbols and logo marks are often presented to viewers as plane figures. It has been recently reported that the perception of plane figures is affected by the psychological influence depending on the layout angles in the field of vision. The subjective geometric relationships of figures are also different from their objective relationships. In our previous research, we examined the relationship between the apparent size of a basic geometric form and its layout angle. When vertices of a square were close to the vertical or horizontal lines, the apparent size of the square seemed to be larger than that of the other layout angles. In this paper, we study how changing the layout angle influences the apparent sizes of various geometric forms and their seeming proportions. Our results show that there are some regular features of the relationship between apparent size and layout angle. Keywords: Apparent Size, Layout Angle, T-Test, Adjustment Method, Aspect Ratio
\end{abstract}

\section{INTRODUCTION}

Many compositional elements such as letters and pictures appear in visual communication media (TV, World Wide Web, newspaper etc.). Symbols and marks that make up the compositional elements in these media appear to the viewer to be plane figures. It has previously been shown that a plane figure has a different psychological influence depending on its layout angle in the field of vision [1]. Kim et al. [2] compared the apparent size of some geometric forms that had the same area. As shown in Figure 1, the figures on the left side seem to be larger than those on the right side. There are clear differences in the apparent sizes of the square and the rhombus; the equilateral triangle and the inverted equilateral triangle; the vertically directed rectangle and the horizontally directed rectangle. This result suggests that the apparent size can vary for the same form or area depending upon the layout angle. Mach [3] pointed out that the apparent size is different for a square when it is positioned on the base of one side and when it is inclined at $45^{\circ}$ (Figure 2). Similarly, it has been reported that the apparent size of a rhombus is larger than that of a square.

In our previous study [4], we examined the apparent size for different layout angles of an equilateral triangle, a square, a pentagon, and an oval. The results showed that the apparent size of the square is the largest compared with the other forms. Moreover, the nearer the layout angles of the apex to the vertical line, the larger the figure's apparent size.

In this study, we investigate the influence of the difference of the layout angle on the apparent size of a rectangle with a different aspect ratio to that of a square.

\section{EXPERIMENT}

\subsection{Stimuli}

Three rectangles ( $\sqrt{2}$ rectangle, golden rectangle, and $\sqrt{4}$ rectangle) were used in the experiment. The area of each form was same, and the layout angle ranged from $15^{\circ}$ to $165^{\circ}$ (Figure 3 ). The color of each figure was assumed to be medium gray. In addition, we performed color measurement with a display color analyzer (KONICA MINOLTA CA-310). Colorimetric values were $\mathrm{x}$ : 0.29, y: 0.29, Lv: 19.02 (These values were indicate by XYZ colormetic system).

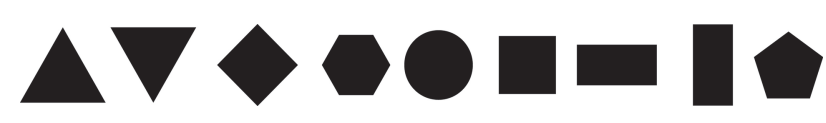

Figure 1: Stimuli in descending order of size.
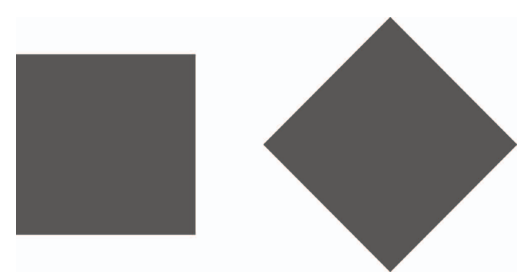

Figure 2: Apparent size of square and rhombus. 


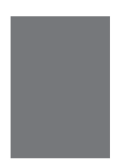

$0^{\circ}$

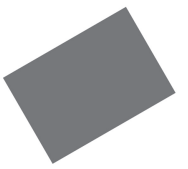

$60^{\circ}$

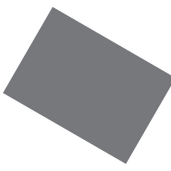

$120^{\circ}$

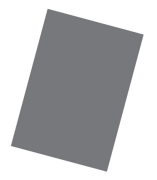

$15^{\circ}$

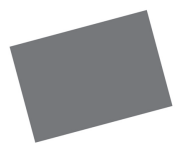

$75^{\circ}$

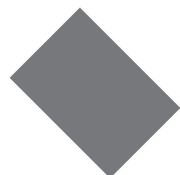

$135^{\circ}$

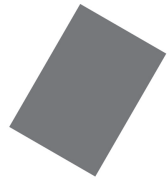

30

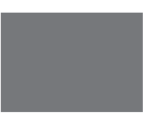

$90^{\circ}$

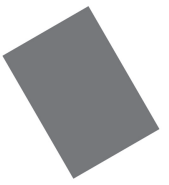

$150^{\circ}$

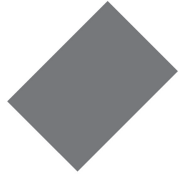

$45^{\circ}$

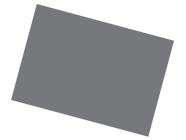

$105^{\circ}$

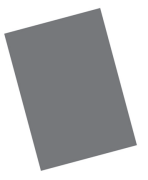

$165^{\circ}$
Figure 3: The Layout angle of $\sqrt{2}$ rectangle stimuli.

\subsection{Method}

Stimuli were presented on a 17-inch color liquid crystal display (NANAO EIZO FlexScan L557-RBK). The resolution was $1,280 \times 1,024$ pixels. The observation distance was about $60 \mathrm{~cm}$ and the eye-level was set at the center of the screen. We fixed participants' heads using a chin rest (Figure 4). Adjusting method was used to compare the apparent size of the forms. For each form, we showed a standard stimulus and several comparison stimuli. The standard stimulus's layout angle was at $0^{\circ}$, and the comparison stimuli's layout angles ranged between $15^{\circ}$ and $165^{\circ}$. All stimuli were displayed in the center of the screen. Each kind of rectangle was presented in randomly ordered blocks, and the orientations in each block were also presented at random. The comparison stimuli were displayed clearly at a larger or smaller size than the standard stimuli. The standard stimuli and the comparison stimuli were set in to a square frame that was invisible to the subjects (Figure 5). The stimuli and the

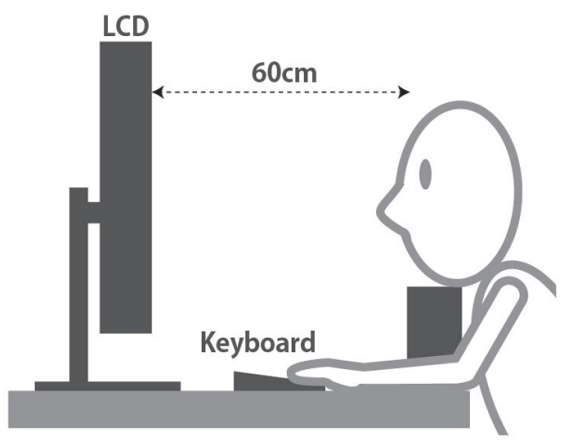

Figure 4: Experimental environment

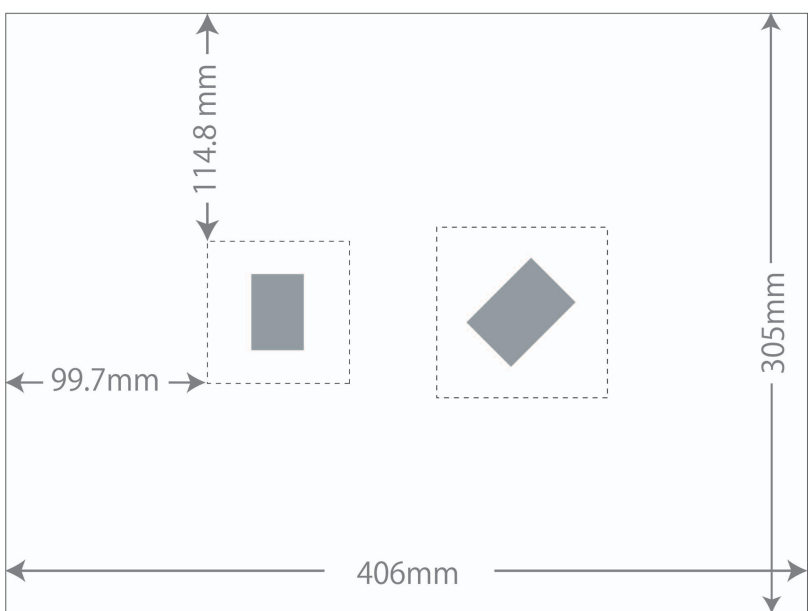

Figure 5: Placement of the standard and comparison stimulus on the screen.

image of square changed size at same ratio. The adjustment program was developed in the Processing 1.2.5 environment. When the right arrow key was pushed, the comparison stimulus's height and width expanded by 1 pixel; when the left arrow key was pushed, it shrank by 1 pixel. Subjects adjusted the size of the comparison stimulus until its apparent size was same as the standard stimulus. The comparison and the standard stimuli were replaced at random positions (right and left). Also, the standard stimuli were presented in random order.

\subsection{Subjects}

40 people (20 males, 20 female) aged from 20 to 24 years with an average age of 22.3 participated in this experiment.

\section{RESULTS}

The size of the comparison stimuli's image was obtained when its apparent size was same as the standard stimulus. The average of the differences was found by subtracting one side of the frame length of the comparison stimuli from the standard stimuli (Figure 6, 7). T-tests were

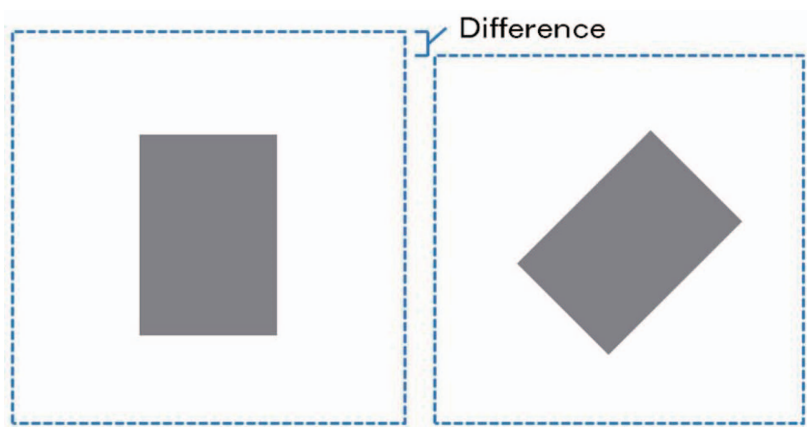

Figure 6: Difference between standard and comparison stimuli. 
performed to reveal differences between the standard and the comparison stimuli on every layout angle for each form (Table 1-3).

For the $\sqrt{2}$ rectangle, the apparent size of the comparison stimuli was largest at $30^{\circ}$ and $135^{\circ}$. The average difference in apparent size between the standard and the comparison stimuli was 10.15 pixels; the result of the t-test was significant $(p<.01)$. In addition, apparent size tended to be larger at $30^{\circ}-60^{\circ}$ and at $120^{\circ}-150^{\circ}$, and smaller at $70^{\circ}-105^{\circ}$.

For the golden rectangle, apparent size was largest at $45^{\circ}$, and the result of the t-test was significant $(p<.01)$. There were significant differences between standard and comparison stimuli: 5.45-7.54 pixels at $30^{\circ}, 60^{\circ}$, and $150^{\circ}(p<.01)$. The smallest difference was 1.4 pixels at $105^{\circ}$, and this difference with the standard stimuli was not significant.

For the $\sqrt{4}$ rectangle, the largest difference was 9.25 pixels at $30^{\circ}(p<.01)$. In addition, even at $135^{\circ}$ and $150^{\circ}$ the apparent size tended to be large. However, at $165^{\circ}$ there were few differences with the standard stimuli. At a placement angle of $60^{\circ}-105^{\circ}$, there were no significant differences with the standard stimuli.

For all three types of rectangle, the apparent size was large at a placement angle of $30^{\circ}, 40^{\circ}$, and $135^{\circ}$ and small when placement angles were $70-105^{\circ}$. Figure 8-1 shows the largest figures, which tended to be longer in the vertical direction. Conversely, Figure 8-2 shows the smallest figures, which tended to be longer in the horizontal direction.

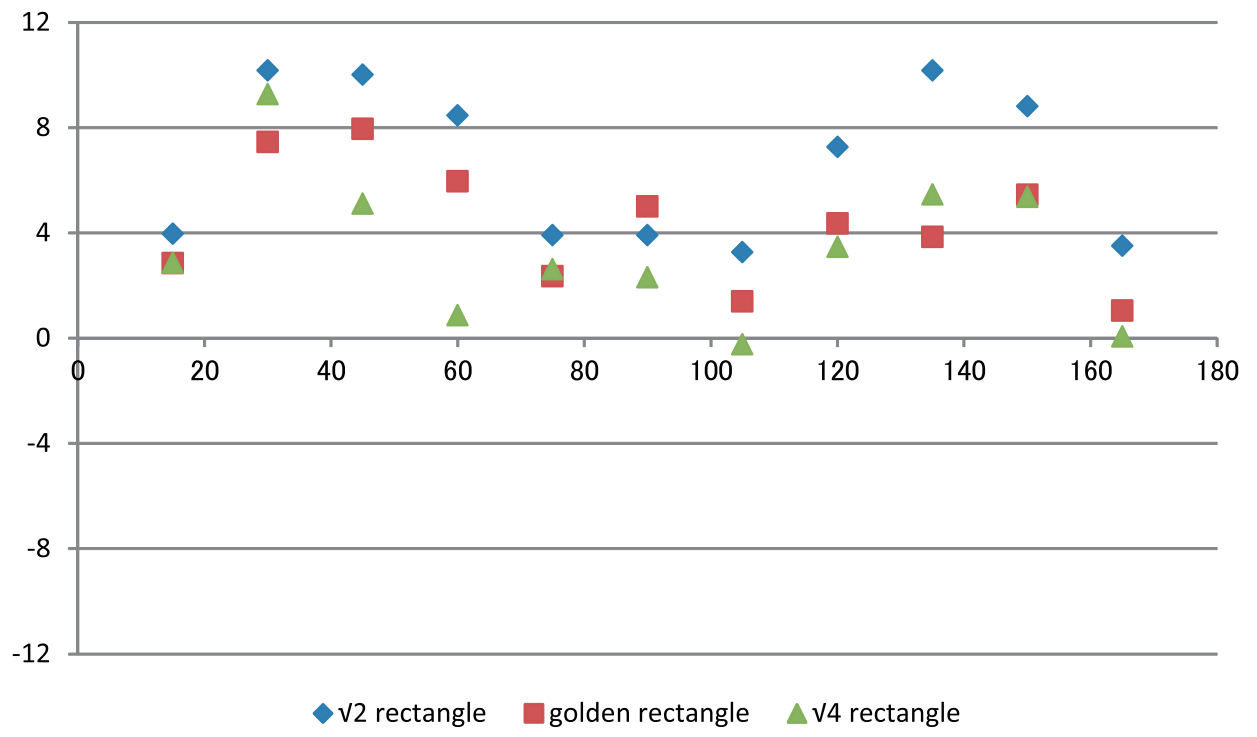

Figure 7: Mean difference between standard and comparison stimuli.

Table 1: T-test results ( $\sqrt{2}$ rectangle $)$.

\begin{tabular}{|c|c|c|c|}
\hline \multicolumn{4}{|c|}{$\sqrt{2}$ rectangle } \\
\hline Angle & Difference & SD & T-test \\
\hline 15 & 3.95 & 9.63421 & $*$ \\
\hline 30 & 10.15 & 8.69291 & $* *$ \\
\hline 45 & 10 & 11.609 & $* *$ \\
\hline 60 & 8.45 & 11.2363 & $* *$ \\
\hline 75 & 3.9 & 11.1948 & $*$ \\
\hline 90 & 3.9 & 16.8672 & \\
\hline 105 & 3.25 & 11.8511 & + \\
\hline 120 & 7.25 & 10.404 & $* *$ \\
\hline 135 & 10.15 & 8.98303 & $* *$ \\
\hline 150 & 8.8 & 9.48737 & $* *$ \\
\hline 165 & 3.5 & 10.0537 & $*$ \\
\hline
\end{tabular}

$* * p<.01, * p<.05,+p<.10$
Table 2: T-test results (golden rectangle).

\begin{tabular}{|c|c|c|c|}
\hline \multicolumn{4}{|c|}{ golden rectangle } \\
\hline Angle & Difference & SD & T-test \\
\hline 15 & 2.85 & 8.88545 & $*$ \\
\hline 30 & 7.45 & 7.89661 & $* *$ \\
\hline 45 & 7.95 & 11.5158 & $* *$ \\
\hline 60 & 5.95 & 9.05241 & $* *$ \\
\hline 75 & 2.35 & 15.2106 & \\
\hline 90 & 5 & 12.6734 & $*$ \\
\hline 105 & 1.4 & 9.7686 & \\
\hline 120 & 4.35 & 13.7366 & + \\
\hline 135 & 3.85 & 9.69681 & $*$ \\
\hline 150 & 5.45 & 10.6986 & $* *$ \\
\hline 165 & 1.05 & 8.31418 & \\
\hline
\end{tabular}

$* * p<.01, * p<.05,+p<.10$ 
Table 3: T-test results ( $\sqrt{4}$ rectangle).

\begin{tabular}{|c|c|c|c|}
\hline \multicolumn{5}{|c|}{$\sqrt{4}$ rectangle } \\
\hline angle & Difference & SD & t-test \\
\hline 15 & 2.85 & 9.42324 & + \\
\hline 30 & 9.25 & 17.6268 & $* *$ \\
\hline 45 & 5.1 & 12.2282 & $*$ \\
\hline 60 & 0.85 & 12.0757 & \\
\hline 75 & 2.6 & 13.0104 & \\
\hline 90 & 2.3 & 15.2756 & \\
\hline 105 & -0.25 & 17.7457 & \\
\hline 120 & 3.45 & 11.5113 & + \\
\hline 135 & 5.45 & 11.2043 & $* *$ \\
\hline 150 & 5.35 & 9.03994 & $* *$ \\
\hline 165 & 0.05 & 10.0816 & \\
\hline
\end{tabular}

$* * p<.01, * p<.05,+p<.10$

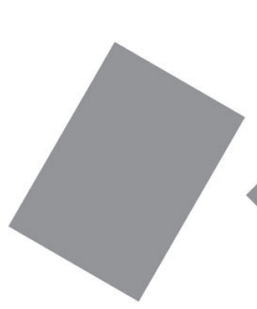

$30^{\circ}$

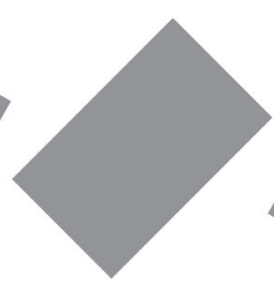

$45^{\circ}$

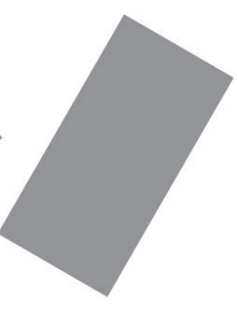

$30^{\circ}$
Figure 8-1: Largest layout angle for each rectangle type.

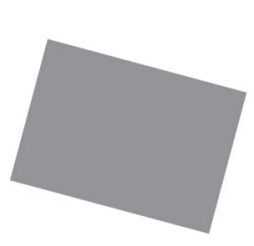

$105^{\circ}$

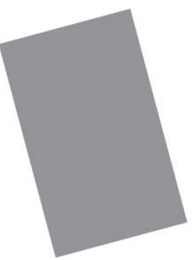

$165^{\circ}$

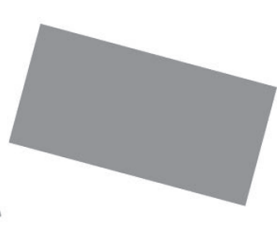

$105^{\circ}$
Figure 8-2: Smallest layout angle for each rectangle type.

\section{DISCUSSION}

In this experiment, we showed that the apparent size of a rectangle changed based on the placement angle. Placement angles at which apparent size was particularly large were $30^{\circ}, 45^{\circ}, 135^{\circ}$, and, $150^{\circ}$ (Figure 7 ). In a previous study, Mach et al. reported that the apparent size of a square was smallest when its placement angle was changed to $45^{\circ}$. Following this result, we compared the apparent size of a square for layout angles that changed every $15^{\circ}$ in our previous study, and found that apparent size was largest at a layout angle of $45^{\circ}$, which accords with the results of this experiment.
When the bisector of the interior angle verged near toward the horizontal and vertical direction, the apparent size tended to be large (Figure 9). It is thought that a special force in the figure influences this tendency. Spatial force is visual psychological effects as influence surrounding space of figure and figure appearance. The special force has influences each apex from the center of the figure [5]. It is thought that this force has influence on the direction of the bisector of the interior angle of the figure (Figure 10). With many stimuli, when the apparent size was large, the bisector of the interior angle tended to be near the horizontal and vertical direction.

The t-test results tended to show a non-significant difference of around $90^{\circ}$. The forms that had the largest difference between the standard stimulus and the comparison stimuli were the $\sqrt{2}$ rectangle, golden rectangle, and $\sqrt{4}$ rectangle in descending order, which accords with the size of the aspect ratio of these three forms. Similarly, apparent size became larger in increasing order of the $\sqrt{2}$ rectangle, golden rectangle, and $\sqrt{4}$ rectangle which accords with aspect ratio of small order. In this case, the bisector of the interior angle at each apex of the figure was close to the opposing corner of the bisector when its apparent size was large and the aspect ratio was small, because the apparent size became large when the distance of the opposing corner of the bisector was near. This result suggests a difference in apparent size occurs more easily when the aspect ratio is small.
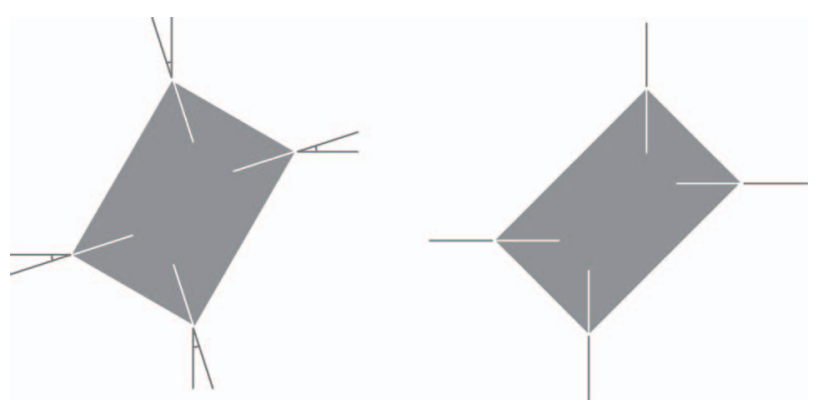

Figure 9: Bisector of the interior angle at each apex of the figure.

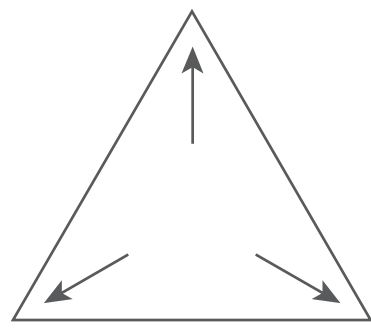

Figure 10: An example of spatial force inside a triangle. 


\section{CONCLUSIONS}

We found that the apparent sizes of three types of rectangle depend greatly upon their layout angle. In particular, the aspect ratio and the half-directional angle of each apex are the important factors that affect the apparent size of the rectangle. That is, the apparent size is large when the aspect ratio is small, and the direction of the layout angle also has some influence.

We believe that the results of this research are basic data and will be useful to apply size adjustment in the design of symbol marks and other compositional elements.

In future studies, we will conduct experiments with other types of geometric forms, and try to examine the spatial force of the figure's outer side.

\section{ACKNOWLEDGEMENTS}

We would like to express our deepest gratitude to $\mathrm{Mr}$. Osa Atsusi, Mr. Matsuda Ken, Mr. Miyake Hiroaki, and Mr. Mizukami Yoshiki. This work was supported by JSPS KAKENHI Grant Number 23611017.

The authors would like to thank Enago (www.enago.jp) for the English language review.

\section{REFERENCES}

1. S. Imai; Sakushi zukei-Miekata no sinrigaku, saiensu sha, p.4 (1984). (in Japanese)

2. H. Kim, et al; Kikagakuzukei no mensekitikaku ni oyobosu eikyou, Journal of Japan Society of Kansei Engineering: 1(1), pp.1-6 (2006). (in Japanese)

3. E. Mach; Kankaku no bunseki, G. Sudo and W. Hiromatu; Housei daigaku syuppankyoku, pp.90-91 (1971). (in Japanese)

4. Y. Fukuda, T. Kinoshita; Effect of Layout Angle Difference of Plane Figure on Apparent Size, Design Research, (59), pp.62-67 (2012).

5. T. Katayama; Iro to Katachi, Manual house, p.58 (2007). (in Japanese)

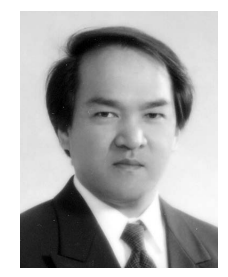

\section{Takeshi KINOSHITA}

Takeshi Kinoshita is an Associate Professor and Advisor to the Vice President, Graduate School of Science and Engineering, Faculty of Engineering, Yamaguchi University since 2000, Yamaguchi Japan, after having attached as a Research Assistant of the Department of Life Science at Yamaguchi Prefectural University since 1995, Yamaguchi Japan, after having attached as a director of Shirogumi Inc. He received Dr. Design in Design Science from Kobe Design University. He is engaged in the design of motion picture (TVCM), and Advertisement design etc. Currently, his researches interests are Design Psychology, Basic Design Education and Kansei engineering. He is now the member of Japan Society of Kansei Engineering, Japanese Society for the Science of Design, and Design Research Association.

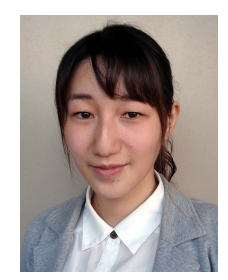

\section{Yumie FUKUDA}

Yumie Fukuda is a student of Graduate School of Science and Engineering, Yamaguchi University. She is engaged in the human visual perception, basic design education, designing a poster and a book cover etc. and teaching graphic software operation. She is now the member of Japan Society of Kansei Engineering, Japan Society For The Science Of Design, and Design Research Association. 